\title{
Autoevaluación de Conocimientos Previos y Rendimiento según Estilos de Aprendizaje en un Grado Universitario de Edificación
}

\author{
Juan M. Alducin-Ochoa y Ana I. Vázquez-Martínez* \\ Universidad de Sevilla, Escuela Técnica Superior de Ingeniería de Edificación, Avda. Reina Mercedes no \\ 4A, 41012 Sevilla - España. (e-mail: alducin@us.es, aisabel@us.es)
}

* Autor a quien debe ser dirigida la correspondencia.

Recibido Sep. 24, 2015; Aceptado Nov. 18, 2015; Versión final Dic. 12, 2015, Publicado Abr. 2016

\begin{abstract}
Resumen
El objetivo fundamental del trabajo fue identificar los estilos de aprendizaje de alumnos de primer curso del grado de Edificación de la Universidad de Sevilla en España. Se estudió la relación existente entre la autoevaluación que hacen los alumnos de sus conocimientos en cuatro materias básicas y las calificaciones, la influencia del estilo en el rendimiento y si existe relación entre las calificaciones obtenidas en materias afines de primer curso del grado. Al efecto, se emplea un diseño metodológico transversal, ex post-facto, utilizando el método descriptivo y correlacional. Se suministró el Cuestionario de Estilos de Aprendizaje de Honey y Alonso (CHAEA), un cuestionario demográfico y de autoevaluación, y se analizaron las calificaciones. Se encontró predominio del estilo reflexivo, que no existe correlación entre autoevaluación y calificaciones, excepto en Matemáticas, y que el comportamiento de los alumnos según los estilos es variable en las distintas materias. Esto indica que para mejorar el rendimiento académico es necesario desarrollar estrategias de clase que den cabida a todos los estilos de aprendizaje.
\end{abstract}

\section{Self-Assessment of Knowledge and Academic Performance according to Learning Styles in a University Degree of Building Construction}

\begin{abstract}
The fundamental objective of this work was to identify the learning styles of first-year students in the degree of Building Construction at the University of Seville in Spain. The existent relationship between student's self-assessment of their own knowledge in four basic courses and their scores, the influence of their learning style on their performance, and whether or not there was a relationship between the scores obtained in related first-year courses were studied. To this end, a cross-sectional methodological design, ex post-facto was used, utilizing correlational and descriptive methods. The Honey-Alonso Learning Styles Questionnaire (CHAEA), a demographic and self-evaluation questionnaire was provided, and the scores analyzed. Predominance of the reflective style and a lack of correlation between self-assessment and scores, except for Mathematics were observed. It was also found that student's behavior according to the styles was variable depending on the different courses. This indicates that, to improve academic performance, the development of in-class strategies that include all learning styles is necessary.
\end{abstract}

Keywords: self-assessment; academic performance; learning styles; grades 


\section{INTRODUCCIÓN}

Los nuevos retos de la Universidad española, vinculados a la Carta Magna de las Universidades Europeas, y la incorporación al Espacio Europeo de Educación Superior sugieren que la enseñanza debe estar basada en las necesidades y peculiaridades individuales. Al respecto, diferentes Declaraciones de la Unión Europea manifiestan la necesidad de introducir vías de aprendizajes flexibles e individualizadas para todos los alumnos. En relación al Espacio Europeo de Educación Superior es necesario destacar que el alumno en este nuevo modelo se convierte en el centro de su aprendizaje, que debe ser activo y participativo, estar intrínsecamente motivado; $y$, de otra parte, el proceso de enseñanza debe ser individualizado.

En el ámbito universitario es importante tomar en consideración los hábitos de estudio y los estilos de aprendizaje de los alumnos para minimizar la deserción escolar. Esta afirmación pone de manifiesto que el punto de partida para diseñar recursos, formatos y actividades realmente eficaces debe ser el conocimiento de las peculiaridades y los estilos de aprendizaje de los alumnos. Este conocimiento permitirá individualizar el proceso de enseñanza-aprendizaje desarrollando métodos docentes acordes a los estilos presentes en el aula y mejorar el aprendizaje y el rendimiento académico. Tomar en consideración los estilos de aprendizaje de los alumnos, y enseñar de acuerdo a los mismos, está relacionado con el rendimiento y satisfacción de los mismos.

Las investigaciones sobre estilos de aprendizaje y rendimiento académico permiten indicar que los alumnos aprenden de formas diferentes, los rendimientos de los alumnos en distintas áreas se relacionan con la forma en que aprenden, enseñándoles según el procedimiento que prefieren el aprendizaje es más efectivo, para identificar las preferencias individuales de aprendizaje se pueden desarrollar procedimientos sistemáticos, y una vez realizado el diagnóstico diseñar prácticas docentes adecuadas.

El objetivo central de esta investigación es determinar los estilos de aprendizaje de los alumnos de primer curso del Grado de Edificación que se imparte en la Universidad de Sevilla, para ello se ha empleado el Cuestionario de Estilos de Aprendizaje Honey y Alonso (2012). Una vez realizado el diagnóstico se abordó el segundo objetivo, que era estudiar el rendimiento en las diferentes asignaturas en función de los estilos dominantes.

La titulación en la que se ha realizado la presente investigación, perteneciente al ámbito de la Arquitectura e Ingeniería, se caracteriza por un bajo rendimiento académico y una alta tasa de deserción. Al igual que en otros estudios e investigaciones, en este también se constata que la mayoría de los alumnos cuando acceden a la titulación presentan dificultades en el aprendizaje de Química, Geometría y Física, bajo nivel en matemáticas, así como en el conocimiento de los principios y procesos que rigen la Ingeniería, e insuficientemente preparados para resolver problemas.

Las asignaturas de primer curso son Materiales I, Fundamentos físicos, Fundamentos matemáticos, Dibujo arquitectónico y Cad, Geometría descriptiva, Introducción a la construcción, Estructuras arquitectónicas I e Historia de la construcción. Para hacer un correcto seguimiento de las materias deben tener conocimientos suficientes en materias básicas como son Matemáticas, Física, Química y Geología, esta situación promueve la formulación del tercer objetivo que es conocer cómo valoran los alumnos sus conocimientos previos en ellas al comenzar el curso, y analizar la relación que existe entre las autoevaluaciones y los resultados académicos.

Y el cuarto objetivo pretende aportar información sobre si existe coherencia en los resultados que alcanzan los alumnos en grupos de materia afines (constructivas, gráficas y científico-tecnológicas), los cuales son denominados binomios de combinación, siendo estos: Materiales I - Introducción a la construcción; Materiales I - Historia de la construcción; Introducción a la construcción - Historia de la construcción, Dibujo arquitectónico y Cad - Geometría descriptiva; Fundamentos matemáticos - Estructuras arquitectónicas I; Fundamentos físicos - Estructuras arquitectónicas I; Fundamentos físicos - Fundamentos matemáticos.

Los objetivos planteados dan lugar a las siguientes hipótesis de investigación formuladas en términos de hipótesis nulas: H10: "No existe relación entre el estilo de aprendizaje dominante y el rendimiento académico"; H20: "No existe correlación entre la autoevaluación de conocimientos previos al ingreso en la Universidad y el rendimiento en las asignaturas correspondientes de primer curso"; y H30: "No existe correlación en el rendimiento de las asignaturas afines de primer curso del grado“.

\section{LOS ESTILOS DE APRENDIZAJE}

Los docentes a través de sus prácticas de aula deben conseguir que los métodos, los materiales y recursos empleados permitan que todos los alumnos puedan aprender y desarrollar toda su capacidad intelectual y 
de aprendizaje; y el conocimiento de los estilos, y la interacción que presentan con los métodos de enseñanza permiten comprender y mejorar el proceso de enseñanza-aprendizaje (Moreno et al., 2014). Para ello, el profesor deberá convertirse en un organizador de los ambientes de aprendizaje adaptándolos a las necesidades y estilos de aprendizaje de los alumnos, facilitando de esta manera el proceso de aprendizaje y el desarrollo de competencias metacognitivas.

Cuanto mayor sea la semejanza entre el estilo de enseñanza del profesor y el de aprendizaje de los alumnos, se produce una mejora en los resultados académicos (Komarraju et al., 2011). Sin embargo, distintas investigaciones evidencian que uno de los errores más frecuentes de los profesores es no tomar en consideración los estilos de aprendizaje de los alumnos. Aunque, en la actualidad hay una clara preocupación por identificar los estilos de aprendizaje, y en consecuencia mejorar los resultados académicos (Komarraju et al., 2011). Pero entre ambos extremos, tienen que producirse cambios metodológicos, situación que no es frecuente en la enseñanza de la Ingeniería (Holvikivi, 2007). Felder y Silverman (1988) indican al respecto que los estilos de aprendizaje de la mayoría de los alumnos son incompatibles con los estilos de enseñanza de sus profesores, no produciéndose por tanto la adaptación del ambiente de aprendizaje a las individuales presentes en el aula. Holvikivi (2007) atribuye este hecho a que los docentes de las Escuelas de Ingeniería no suelen tener formación pedagógica. Deberá ser preocupación de los docentes ayudar a los alumnos a que desarrollen al máximo el estilo de aprendizaje dominante, el preferido, para conseguir el máximo de sus capacidades (Ventura, 2013), a aprender en cualquier situación (Alonso et al., 2012), a comprender y transformar la experiencia para alcanzar el conocimiento.

Conviene que se establezca, aunque brevemente, qué se entiende por estilo de aprendizaje. Se puede indicar que es la variación individual de los modos de percibir, recordar, pensar, las formas diferentes de aprender, almacenar, transformar y emplear la información (Gentry y Helgesen, 1999), cómo los alumnos aprenden en un contexto social (Hamidah et al., 2009), la forma preferida de aprendizaje de un individuo (Alonso et al., 2012), o el conjunto de preferencias personales para hacer algo y que se manifiesta a través de patrones conductuales que lo hacen diferentes de los demás (Alonso et al., 2012).

En cuanto a los factores que influyen en el estilo de aprendizaje, Kolb y Kolb (2005) consideran que el estilo de aprendizaje está condicionado por factores hereditarios, experiencias previas y las características del ambiente en el que se desarrolla, y ello hace que cada uno presente maneras diferentes de aprender. De otra parte, el estilo de aprendizaje de los alumnos, así como las estrategias seguidas, puede variar en función de la percepción o experiencia de aprendizaje en cada asignatura que curse, e incluso en el estudio de temas diferentes dentro de cada materia (Ventura, 2013). Considerando, además, que conforme el estudiante progresa en su proceso de formación, el estilo se vuelve más avanzado y maduro (AbdulRahman y Boulay, 2014), y esto puede deberse a que los alumnos se hayan adaptado cognitivamente al ámbito universitario y a las estrategias empleadas por los docentes (Acevedo et al., 2015b).

Con carácter general a los estilos se le pueden atribuir las siguientes características: cada persona tiene un estilo de aprendizaje predominante; los estilos de aprendizaje existen en un continuo, aunque en ocasiones sean descritos como opuestos; cada estilo tiene un valor neutro, ninguno es mejor o peor que otro; el profesor debe propiciar que los alumnos alcancen consciencia de sus estilos de aprendizaje preferentes; los estilos de aprendizaje son flexibles; y las personas, como los estilos, se desarrollan y transforman con el tiempo. Para la identificación de los estilos, en esta investigación se ha empleado el Cuestionario de Estilos de Aprendizaje Honey y Alonso, CHAEA (2012), instrumento empleado con gran éxito en la comunidad latinoamericana (Ventura, 2013).

\section{MATERIALES Y MÉTODO}

\section{Participantes}

Esta investigación se desarrolló en la Escuela Técnica Superior de Ingeniería de Edificación (Universidad de Sevilla) en la que se imparte el Grado de Edificación, con alumnos matriculados en primer curso. La muestra para el estudio estuvo constituida por 161 alumnos, de los cuales 59 (36.65\%) son mujeres y 102 (63.35\%) hombres. Por edad, la distribución es 108 (67.08\%) alumnos entre 18-20 años, 34 (21.12\%) 21-25 años, 13 (8.07\%) entre 26-30 años, y 6 (3.73\%) mayores de 30 años, siendo la media de 20.72 años.

\section{Instrumentos}

Ya se ha indicado que el instrumento para la determinación de los estilos de aprendizaje ha sido el cuestionario CHAEA, que consta de 80 ítems, 20 por cada uno de los estilos que analiza, las posibles respuestas se presentan de manera dicotómica (+ 0 -) en función de si se está de acuerdo con la proposición del ítem (+) o en desacuerdo (-). Y con él se pretenden identificar cuatro estilos: activo, reflexivo, teórico y pragmático. En la tabla 1 se exponen algunas de las características de cada estilo (Alonso et al., 2012). 
Tabla 1: Algunas características de los estilos de aprendizaje

\begin{tabular}{|l|l|}
\hline $\begin{array}{l}\text { Estilo activo: } \\
\text { creativo, animador, novedoso, improvisador, } \\
\text { descubridor, arriesgado, inventor, espontáneo, vital, } \\
\text { generador de ideas, protagonista, innovador, } \\
\begin{array}{l}\text { conversador, líder, voluntarioso, participativo, } \\
\text { competitivo, deseoso de aprender, solucionador de } \\
\text { problemas, cambiante. }\end{array}\end{array}$ & $\begin{array}{l}\text { Estilo reflexivo: } \\
\text { observador, ponderado, concienzudo, recopilador, } \\
\text { receptivo, analítico, paciente, exhaustivo, cuidadoso, } \\
\text { elaborador de argumentos, previsor de alternativas, } \\
\text { estudioso de comportamientos, investigador, asimilador, } \\
\text { escritor de informes y/o declaraciones, lento, distante, } \\
\text { prudente. }\end{array}$ \\
\hline $\begin{array}{l}\text { Estilo teórico: } \\
\text { metódico, estructurado, objetivo, planificado, crítico, } \\
\text { disciplinado, sintético, razonador, lógico, pensador, } \\
\text { perfeccionista, generalizador, buscador de hipótesis, } \\
\text { teorías y modelos, interesado en el "por qué", inventor } \\
\text { de procedimientos. }\end{array}$ & $\begin{array}{l}\text { Estilo pragmático: } \\
\text { técnico, experimentador, práctico, eficaz, directo, } \\
\text { realista, rápido, decidido, planificador, positivo, concreto, } \\
\text { objetivo, claro, seguro de sí, solucionador de problemas, } \\
\text { aplicador de lo aprendido, planificador de acciones. }\end{array}$ \\
\hline
\end{tabular}

La fiabilidad del cuestionario CHAEA se ha determinado recurriendo al coeficiente alfa de Cronbach, obteniéndose un valor $\alpha=.7275$, que indica según George y Mallery (1995) un nivel aceptable de fiabilidad. Para adscribir a los alumnos a un estilo de aprendizaje concreto se ha seguido el criterio de emplear la puntuación máxima individual, sin situarlos en comparación la muestra, criterio seguido por diferentes autores, como Gil Madrona et al., (2007), Gravini (2008), entre otros.

Los datos de la autoevaluación que hacen los alumnos de sus conocimientos en Geología, Química, Física y Matemáticas fueron recogidos con el Cuestionario demográfico y de autoevaluación (CDAT), en el que, además de los aspectos demográficos, se solicita a los alumnos que valoren de 0 a 10 los conocimientos que creen tener en las materias citadas. El rendimiento académico de los alumnos se obtiene de las actas oficiales de la primera convocatoria ordinaria de las asignaturas de primer curso de la titulación, facilitadas por la secretaría del centro. Los cuestionarios CHAEA y CDAT se suministraron durante la primera semana de curso, y las calificaciones se obtienen al finalizar el periodo de exámenes y elaboración de las actas de las asignaturas.

\section{Método}

Los objetivos de investigación planteados sugieren un diseño metodológico transversal, ex post facto con variables no manipuladas experimentalmente, empleando el método descriptivo y el correlacional.

\section{Análisis de los datos}

Se ha empleado el paquete informático SPSS versión 17. Para la determinación de los estilos dominantes se ha realizado un estudio descriptivo univariante. Para dar respuesta a las hipótesis de investigación se empleará el coeficiente de correlación de Pearson, estableciéndose un nivel de confianza del $95 \%(\alpha=.05)$.

\section{RESULTADOS}

Estilos de aprendizaje hallados: Las puntuaciones medias en cada uno de los estilos, ordenadas de mayor a menor, son: Reflexivo $(M=16.02 ; S D=2.671)$, Pragmático $(M=13.54 ; S D=2.324)$, Teórico $(M=13.23$; $S D=2.448)$ y Activo $(M=11.11 ; S D=3,197)$. La distribución de estilos dominantes por sexo se recoge en la tabla 2, en ella queda reflejado que el porcentaje de mujeres reflexivas es superior al de hombres reflexivos, mientras que el porcentaje de hombres activos y pragmáticos es superior al de mujeres respectivamente.

Tabla 2: Distribución de Estilos de Aprendizaje dominantes por sexo

\begin{tabular}{|l|c|c|c|c|c|}
\hline \multirow{2}{*}{} & \multicolumn{2}{|c|}{ Mujeres } & \multicolumn{2}{c|}{ Hombres } & \multirow{2}{*}{ Total } \\
\cline { 2 - 5 } & frecuencia & $\%$ & frecuencia & $\%$ & 12 \\
\hline Estilo Activo & 4 & 6.78 & 8 & 7.84 & 101 \\
\hline Estilo Reflexivo & 42 & 71.19 & 59 & 57.84 & 5 \\
\hline Estilo Teórico & 2 & 3.39 & 3 & 2.94 & 20 \\
\hline Estilo Pragmático & 5 & 8.47 & 15 & 14.71 & 23 \\
\hline Multiestilo & 6 & 10.17 & 17 & 16.67 & 161 \\
\hline Total & 59 & 100.00 & 102 & 100.00 & 1 \\
\hline
\end{tabular}


La tabla 2 también pone de manifiesto que hay alumnos que presentan combinación de estilos, es decir, presentan el mismo puntaje en dos o más estilos, a los que se denomina multiestilo. Las combinaciones detectadas en la muestra son: reflexivo-teórico $(f=7 ; 4,35 \%)$, reflexivo-pragmático $(f=6 ; 3,73 \%)$, activoreflexivo $(f=4 ; 2,48 \%)$, teórico-pragmático $(f=2 ; 1,24 \%)$, activo-reflexivo-pragmático $(f=2 ; 1,24 \%)$, activopragmático $(f=1 ; 0,62 \%)$, y reflexivo-teórico-pragmático $(f=1 ; 0,62 \%)$.

Rendimiento por estilo de aprendizaje dominante: En la tabla 3 se presentan los estadísticos descriptivos de las calificaciones en las asignaturas en función del estilo de aprendizaje dominante.

Tabla 3: Descriptivos de las calificaciones en las asignaturas por estilo de aprendizaje dominante

\begin{tabular}{|l|c|c|c|c|c|c|c|c|c|c|c|c|}
\hline \multirow{2}{*}{ Asignaturas } & \multicolumn{3}{|c|}{ Activo $(N=12)$} & \multicolumn{3}{c|}{ Reflexivo $(N=101)$} & \multicolumn{3}{c|}{ Teórico $(N=5)$} & \multicolumn{3}{c|}{ Pragmático $(N=20)$} \\
\cline { 2 - 15 } & $f$ & $M$ & $S D$ & $f$ & $M$ & $S D$ & $f$ & $M$ & $S D$ & $f$ & $M$ & $S D$ \\
\hline Materiales I & 10 & 4.22 & 2.658 & 68 & 5.32 & 2.536 & 3 & 6.37 & 2.491 & 15 & 3.51 & 2.495 \\
\hline Física & 8 & 4.59 & 1.376 & 45 & 5.15 & 1.624 & 2 & 6.40 & 1.273 & 9 & 6.64 & 1.751 \\
\hline Matemáticas & 4 & 5.50 & 1.970 & 43 & 5.68 & 1.849 & 3 & 7.33 & 1.528 & 9 & 5.03 & 2.992 \\
\hline Dibujo & 8 & 5.56 & 0.729 & 59 & 5.34 & 1.555 & 2 & 6.50 & 0.707 & 11 & 5.41 & 1.020 \\
\hline Descriptiva & 5 & 6.36 & 1.906 & 44 & 6.05 & 1.504 & 0 & & & 12 & 6.53 & 1.789 \\
\hline Construcción & 9 & 4.23 & 1.218 & 66 & 5.35 & 1.772 & 4 & 5.48 & 1.445 & 15 & 4.85 & 2.611 \\
\hline Estructuras I & 8 & 5.25 & 1.482 & 64 & 6.72 & 1.328 & 3 & 6.57 & 1.222 & 13 & 6.40 & 1.729 \\
\hline Historia & 8 & 6.19 & 1.280 & 64 & 7.28 & 1.434 & 3 & 7.67 & 1.443 & 14 & 6.87 & 1.711 \\
\hline
\end{tabular}

En el estilo activo, la asignatura que registra el mayor número de alumnos presentados es "Materiales I" $(f=10)$, seguida de "Construcción" $(f=9)$, "Historia" $(f=8)$, "Estructuras I" $(f=8)$, "Física" $(f=8)$, "Dibujo" $(f=8)$, y las que registran el menor número de alumnos presentados son "Descriptiva" $(f=5)$ y "Matemáticas" $(f=4)$. Los estadísticos del estilo activo indican que "Descriptiva" obtiene la media más alta $(M=6.36)$, seguida por "Matemáticas" $(M=5.50)$. Por el contrario, en "Materiales I" se encuentra la media más baja $(M=4.22)$ y con las calificaciones más dispersas $(S D=2.658)$. Las calificaciones más homogéneas se encuentran en "Dibujo" $(S D=0.729)$.

Para el estilo Reflexivo, es la asignatura "Materiales I" ( $f=68)$ la que cuenta con mayor número de alumnos presentados, seguida de "Construcción" $(f=66)$, "Historia" $(f=64)$, "Estructuras I" $(f=64)$, "Dibujo" $(f=59)$, siendo las que registran el menor número de alumnos presentados "Física" $(f=45)$, "Descriptiva" $(f=44)$ y "Matemáticas" $(f=43)$. Los estadísticos de este estilo indican que en "Historia" se obtiene la media más alta $(M=7.28)$ con unas calificaciones muy homogéneas $(S D=1.434)$, aunque las calificaciones más homogéneas corresponden a "Estructuras I" ( $S D=1.328)$. Por el contrario, la media más baja se encuentran en "Física" $(M=5.15)$ y las más dispersas en "Materiales I" ( $S D=2.536)$.

Respecto al estilo Teórico, es la asignatura de "Construcción" $(f=4)$ la que cuenta con un mayor número de presentados, seguida de "Materiales I" $(f=3)$, "Historia" $(f=3)$, "Estructuras I" $(f=3)$, "Matemáticas" $(f=3)$, y "Física" ( $f=2)$, "Dibujo" $(f=2)$ y "Descriptiva" $(f=0)$ las que cuentan con el menor número de alumnos presentados. Los estadísticos indican que "Historia" obtiene la media más alta $(M=7.67)$ y "Construcción" la más baja $(M=5.48)$.

En el estilo Pragmático, las asignaturas con mayor número de presentados son "Materiales l" y "Construcción" ( $f=15)$, seguidas de "Historia" $(f=14)$, "Estructuras I" $(f=13)$, "Descriptiva" $(f=12)$, y las que registran el menor número de alumnos presentados son "Dibujo" $(f=11)$ y "Matemáticas" y "Física" $(f=9)$. Los estadísticos del estilo pragmático informan que "Historia" obtiene la media más alta $(M=6.87)$ y "Materiales I" la más baja $(M=3.51)$. Las calificaciones más homogéneas se dan en "Dibujo" $(S D=1.020)$, y las más dispersas en "Matemáticas" $(S D=2.992)$.

La Figura 1 presenta las medias de las calificaciones en las asignaturas de primer curso del grado para los estilos de aprendizaje dominantes activo, reflexivo y pragmático, dejando sin representar el estilo teórico al ser la frecuencia de alumnos en este estilo muy pequeña ( $f<5$ como refleja la tabla 3$)$. En esta figura se observa que en las asignaturas "Matemáticas", "Dibujo" y "Descriptiva" la diferencia de las notas medias para los estilos activo, reflexivo y pragmático es muy pequeña; esta situación también se da en las asignaturas de "Construcción" e "Historia" para los estilos reflexivo y pragmático, siendo algo mayor la diferencia con las del estilo activo. 
En la asignatura de "Física" se aprecia que la diferencia de la nota media del estilo pragmático es elevada con respecto a las de los estilos reflexivo y activo donde las notas medias son más bajas (ver Fig. 1), siendo estas diferencias significativas $F(2,59)=4.090, p=.022$, con tamaño de efecto medio $R^{2}=.12$, confirmándose mediante la prueba post hoc DMS (diferencias mínimas significativas), que las diferencias significativas se encuentran entre el activo y pragmático $(p=.011)$ y entre el reflexivo y pragmático $(p=.014)$. En la asignatura "Estructuras I" la diferencia de las notas medias para los estilos reflexivo y pragmático es elevada respecto a la del estilo activo donde la nota media es más baja (ver Fig. 1), resultando significativas las diferencias al aplicar, por no cumplirse el supuesto de normalidad, la prueba no paramétrica de Kruskal-Wallis, $X^{2}(2, N=85)=6.386, p=.041$. Por último, en la asignatura "Materiales I" la diferencia de las notas medias para los tres estilos es apreciable tal como se observa en la figura 1, resultando significativas las diferencias al aplicar, por no cumplirse tampoco el supuesto de normalidad, la prueba no paramétrica de Kruskal-Wallis, $X^{2}(2, N=93)=7.038, p=.030$.

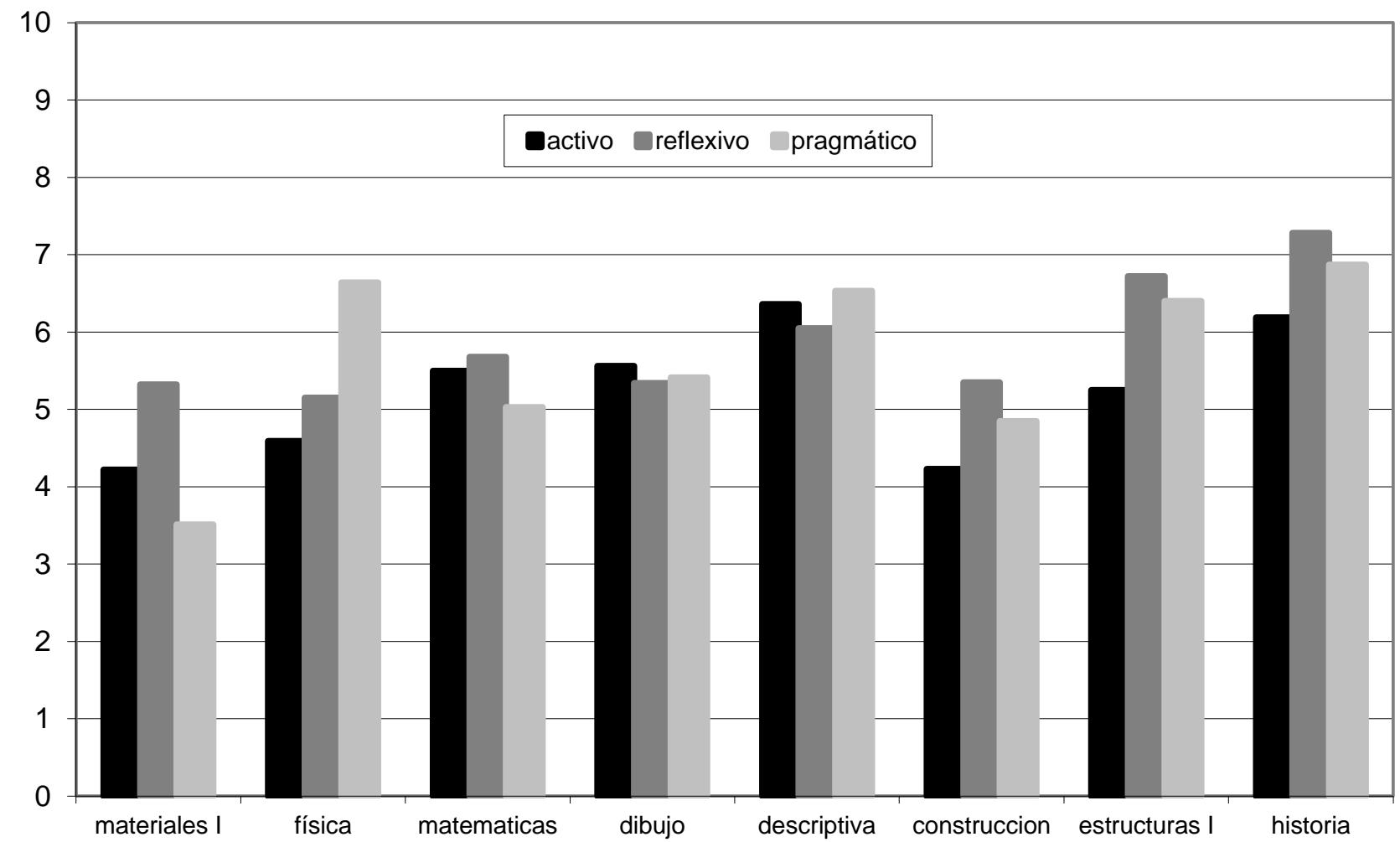

Fig. 1: Media de calificaciones en asignaturas por estilo de aprendizaje dominante.

\section{Relación entre autoevaluación de conocimientos y rendimiento en asignaturas afines}

Se pretende determinar la posible correlación entre la Autoevaluación de conocimientos previos en Química y Geología con la asignatura de "Materiales I", Física en relación con las asignaturas "Física" y "Estructuras I", y Matemáticas en relación con las asignaturas "Matemáticas" y "Estructuras I". Los valores de correlación de Pearson se recogen en la tabla 4.

Tabla 4: Correlación de Pearson para los binomios de combinación en la muestra. ** Correlación significativa al nivel .01 (bilateral).

\begin{tabular}{|c|c|c|c|}
\hline Binomios de combinación & $N$ & $r_{x, y}$ & Sig. \\
\hline Autoevaluación Geología - Materiales I & 114 & .180 & .055 \\
\hline Autoevaluación Química - Materiales I & 114 & .141 & .134 \\
\hline Autoevaluación Física - Física & 76 & .211 & .068 \\
\hline Autoevaluación Física - Estructuras I & 108 & .072 & .461 \\
\hline Autoevaluación Matemáticas - Matemáticas & 71 & $.315^{\star \star}$ & .008 \\
\hline Autoevaluación Matemáticas - Estructuras I & 108 & .083 & .393 \\
\hline
\end{tabular}


Se observa que ningún binomio de correlación presenta resultados significativos, excepto entre la autoevaluación que hacen los alumnos de sus conocimientos en matemáticas y la nota que obtienen en la asignatura "Matemáticas", que presenta correlación positiva estadísticamente significativa $r(N=71)=.315$, $p=.008$, con un tamaño de efecto medio. Profundizando en el estudio de este binomio significativo, en la tabla 5 se presentan los valores de correlación de Pearson diferenciando por estilo de aprendizaje dominante.

Tabla 5: Correlación de Pearson para el binomio significativo por estilos de aprendizaje dominante. * Correlación significativa al nivel .05 (bilateral); ${ }^{* *}$ Correlación significativa al nivel .01 (bilateral)

\begin{tabular}{|l|c|c|c|c|c|}
\hline Binomio de combinación & Activo & Reflexivo & Teórico & Pragmático & Multiestilo \\
\hline Autoevaluación Matemáticas - Matemáticas & $r=-.982^{*}$ & $r=.398^{* *}$ & $r=-.143$ & $r=.505$ & $r=.405$ \\
& $N=4$ & $N=43$ & $N=3$ & $N=9$ & $N=12$ \\
& $p=.018$ & $p=.008$ & $p=.909$ & $p=.165$ & $p=.192$ \\
\hline
\end{tabular}

Estos resultados permiten ratificar que la correlación hallada entre la autoevaluación de matemáticas y la calificación en "Matemáticas" es debida a los alumnos reflexivos en los que se encontró correlación positiva estadísticamente significativa $r(N=43)=.398, p=.008$, con un tamaño de efecto medio, mientras que en los alumnos teóricos y pragmáticos no hay correlación significativa, y aunque se hubiese encontrado en los alumnos teóricos tampoco se les podría atribuir su influencia al ser muy pequeña la frecuencia de este estilo $(f=3)$. Y por otra parte, en los alumnos activos se logra correlación negativa significativa, que tampoco es relevante al ser también muy pequeña la frecuencia de este estilo $(f=4)$.

\section{Relación del rendimiento entre asignaturas afines}

Se aborda a continuación la posible relación entre las calificaciones de asignaturas que se entienden afines, bien por estar relacionadas con la Construcción (Materiales I - Introducción a la construcción - Historia de la construcción), por pertenecer al área gráfica (Dibujo - Descriptiva) o al área científico-tecnológica (Física - Matemáticas - Estructuras). Los valores de correlación de Pearson se recogen en la tabla 6.

Tabla 6: Correlación de Pearson para los binomios de combinación. * Correlación significativa al nivel .05 (bilateral); ${ }^{* \star}$ Correlación significativa al nivel .01 (bilateral)

\begin{tabular}{|l|c|c|c|}
\hline Binomios de combinación & $N$ & $r_{x, y}$ & Sig. \\
\hline Materiales I - Construcción & 95 & $.202^{*}$ & .050 \\
\hline Materiales I - Historia & 92 & $.462^{* *}$ & .000 \\
\hline Construcción - Historia & 101 & -.081 & .423 \\
\hline Dibujo - Descriptiva & 63 & $.361^{* *}$ & .004 \\
\hline Matemáticas - Estructuras I & 63 & $.297^{*}$ & .018 \\
\hline Física - Estructuras I & 67 & $.332^{* *}$ & .006 \\
\hline Física - Matemáticas & 52 & $.712^{* *}$ & .000 \\
\hline
\end{tabular}

Se ha encontrado correlación positiva estadísticamente significativa en las combinaciones: "Materiales I Construcción" $r(N=95)=.202, p=.050$, con un tamaño de efecto pequeño; el tamaño de efecto medio se ha hallado en los binomios "Materiales I - Historia" $r(N=92)=.462, p=.000$, "Dibujo - Descriptiva" $r(N=63)=.361$, $p=.004$, "Matemáticas - Estructuras I" $r(N=63)=.297, p=.018$, y "Física - Estructuras I" $r(N=67)=.332$, $p=.006$; y con tamaño de efecto grande el binomio "Física - Matemáticas" $r(N=52)=.712, p=.000$.

Profundizando en el estudio de los binomios con diferencias significativas, en la tabla 7 se presentan los valores de correlación de Pearson diferenciando por estilo de aprendizaje dominante. Estos resultados ponen de manifiesto ciertos aspectos de las correlaciones anteriormente encontradas: a) la encontrada en el binomio "Materiales I - Historia" se debe a la correlación positiva estadísticamente significativa hallada en los alumnos reflexivos $r(N=55)=.381, p=.004$ con un tamaño de efecto medio, en los pragmáticos $r(N=13)=.826, p=.000$ con un tamaño de efecto grande, y en los que presentan combinación de estilos $r(N=16)=.608, p=.013$ con un tamaño de efecto grande; b) la encontrada en el binomio "Dibujo Descriptiva" se debe a la correlación positiva estadísticamente significativa hallada en los alumnos reflexivos $r(N=36)=.561, p=.000$ con un tamaño de efecto grande; c) la encontrada en el binomio "Física Matemáticas" se debe a la correlación positiva estadísticamente significativa hallada en los alumnos reflexivos $r(N=32)=.716, p=.000$ con un tamaño de efecto grande. Como se observa, ni los alumnos teóricos 
ni los activos influyen en las correlaciones encontradas con todos los alumnos de la muestra, aunque si influyeran, tampoco se les podría atribuir al ser muy pequeña la frecuencia de alumnos de estos estilos. Por otra parte, en los binomios "Materiales I - Construcción", "Matemáticas - Estructuras I" y "Física Estructuras I" que dieron correlación significativa al trabajar con todos los alumnos, cuando se separa el análisis por estilo de aprendizaje dominante, se pierde la correlación no pudiéndose atribuírsela a ningún estilo de aprendizaje dominante.

Tabla 7: Correlación de Pearson para los binomios significativos por estilos de aprendizaje dominante. * Correlación significativa al nivel .05 (bilateral); ${ }^{* *}$ Correlación significativa al nivel .01 (bilateral); a. No se puede calcular al no existir datos en una variable

\begin{tabular}{|l|c|c|c|c|c|}
\hline Binomio de combinación & Activo & Reflexivo & Teórico & Pragmático & Multiestilo \\
\hline Materiales I - Construcción & $r=-.082$ & $r=.130$ & $r=.957$ & $r=.267$ & $r=.179$ \\
& $N=8$ & $N=55$ & $N=3$ & $N=14$ & $N=15$ \\
& $p=.847$ & $p=.345$ & $p=.188$ & $p=.356$ & $p=.523$ \\
\hline Materiales I - Historia & $r=-.127$ & $r=.381^{* *}$ & $r=-1.0$ & $r=.826^{* *}$ & $r=.608^{*}$ \\
& $N=6$ & $N=55$ & $N=2$ & $N=13$ & $N=16$ \\
& $p=.811$ & $p=.004$ & $p=$. & $p=.000$ & $p=.013$ \\
\hline Dibujo - Descriptiva & $r=.647$ & $r=.561^{* *}$ & a & $r=.411$ & $r=-.002$ \\
& $N=4$ & $N=36$ & $N=0$ & $N=9$ & $N=14$ \\
& $p=.353$ & $p=.000$ & $p=$. & $p=.272$ & $p=.996$ \\
\hline Matemáticas - Estructuras I & $r=-.027$ & $r=.192$ & $r=.786$ & $r=.683$ & $r=.087$ \\
& $N=3$ & $N=37$ & $N=3$ & $N=8$ & $N=12$ \\
& $p=.983$ & $p=.255$ & $p=.425$ & $p=.062$ & $p=.787$ \\
\hline Física - Estructuras I & $r=.394$ & $r=.262$ & $r=1.0$ & $r=.444$ & $r=.485$ \\
& $N=6$ & $N=40$ & $N=2$ & $N=7$ & $N=12$ \\
& $p=.439$ & $p=.103$ & $p=$. & $p=.318$ & $p=.110$ \\
\hline Física - Matemáticas & $r=.764$ & $r=.716^{* *}$ & $r=1.0$ & $r=.589$ & $r=.926^{* *}$ \\
& $N=4$ & $N=32$ & $N=2$ & $N=6$ & $N=8$ \\
& $p=.236$ & $p=.000$ & $p=$. & $p=.219$ & $p=.001$ \\
\hline
\end{tabular}

\section{DISCUSIÓN}

Los resultados obtenidos en el orden de mayor a menor promedio de puntuación en las escalas de los estilos de aprendizaje (reflexivo-pragmático-teórico-activo), coinciden con los hallados en otras investigaciones en el ámbito latinoamericano en el área de Arquitectura e Ingeniería (Acevedo y Rocha, 2011). Los resultados alcanzados no coinciden con otros, entre los que se destaca aquellos en los que el ordenamiento es reflexivo-teórico-pragmático-activo (Craveri y Anido, 2009), los que el orden hallado es reflexivo-pragmático-activo-teórico (Acevedo et al., 2015a), aquellos que concluyen con un claro predominio del estilo pragmático (Bolívar y Rojas, 2008); los de orden pragmático-reflexivo-teórico-activo (Villamizar, 2012), los que obtienen un predominio del estilo activo seguido del reflexivo (Ramos y Triana, 2011), 0 preferencia por los estilos teórico y reflexivo (Escalante et al., 2009).

En cuanto a la influencia del sexo en el estilo de aprendizaje se ha obtenido que las alumnas son más reflexivas que los alumnos y estos más pragmáticos que ellas, estos resultados coinciden con otros realizados en el ámbito de la Ingeniería y de las Ciencias (Acevedo et al., 2015a). Y difiere de otros, en los que no se encuentran diferencias entre hombres y mujeres (Ventura et al., 2014).

De otra parte, excepto en la autoevaluación de matemáticas realizada por los alumnos del estilo reflexivo y la calificación obtenida en la asignatura de Matemáticas, en el análisis de la autoevaluación que realizan los alumnos de sus conocimientos y la calificación que luego obtienen en las asignaturas ha quedado patente que no existe correspondencia entre lo que el alumno considera que sabe y la calificación que obtiene. Esto supone que los docentes deben ayudar a los alumnos a que realmente sean capaces de valorar de manera objetiva los recursos personales de los que dispone, aspecto característico de las personas reflexivas, pero que solo se produjo en el área de las Matemáticas.

Relativo a la relación entre estilos y rendimiento académico en las diferentes asignaturas, los resultados hallados son que los alumnos de estilo reflexivo son los que obtienen las medias más altas en Estructuras-I, 
los pragmáticos en Física y Descriptiva, y en el resto de asignaturas lo hacen los alumnos teóricos. Estos resultados no coinciden con los de otras investigaciones, como la de Morales-Ramírez et al. (2012) que obtienen que las mejores calificaciones la consiguen los alumnos reflexivos seguidos de los pragmáticos, la de Cantú (2004) que no encuentra relación entre estilo y rendimiento en la asignatura de diseño, la de Demirbas y Demirkan (2007) quienes concluyen que los alumnos que obtienen las mejores calificaciones en Dibujo son los alumnos pragmáticos; en Matemáticas coincide con Luengo y González (2005) en la que alcanzan las mejores notas los estilos teóricos seguidos de los reflexivos, pero difiere de Gallego y Nevot (2008) quienes indican un mejor rendimiento para alumnos reflexivos y con la de Orhun (2012) que hace lo propio con el estilo pragmático.

En las calificaciones obtenidas en materias afines se ha hallado que las notas obtenidas en la asignatura "Materiales I" guarda relación con las obtenidas en la asignatura "Historia de la construcción" e "Introducción a la construcción", todas del área de conocimiento "Construcciones arquitectónicas"; las de "Dibujo arquitectónico y Cad" con "Geometría descriptiva", ambas del área de Expresión gráfica; y las de "Fundamentos físicos", "Fundamentos matemáticos" y "Estructuras arquitectónicas I" guardan relación entre ellas, perteneciendo todas al área científico-tecnológica. Correlaciones cimentadas esencialmente por los alumnos del estilo reflexivo y pragmático.

Es de destacar la importancia de la presencia del estilo reflexivo, dado que diferentes estudios han demostrado que los alumnos que presentan este estilo tienen una mayor predisposición hacia la metacognición (Arias et al., 2014), es decir, son alumnos con capacidad para evaluar sus aprendizajes y los procesos que guían su consecución. Sin embargo, y tomando en consideración la investigación de Safarin et al. (2013) lo ideal es que los alumnos de Ingeniería presentasen un predominio multiestilo reflexivopragmático. Al respecto Escalante et al. (2009) advierten de la necesidad de formar a los alumnos de Ingeniería en una variedad de estilos, para en primer lugar transformarlos en alumnos eficaces y posteriormente facilitarles la adaptación a la vida profesional.

Ventura et al. (2014) indican que cada alumno debería estar formado en más de un estilo, aunque uno tuviera más peso que otro. En este sentido se debe tomar en consideración la relación entre estilos y funciones del Ingeniero expuesta por Gravini (2008), así el estilo teórico será especialmente útil cuando deba conjugar avances tecnológicos-necesidades del sector-necesidades locales; el reflexivo cuando lo que destaque sea la capacidad de análisis para proponer soluciones a los problemas tomando en consideración todos los factores de influencia; deberá ser activo para poner en funcionamiento nuevos proyectos o empresas y pragmático para localizar y aplicar los diversos indicadores que acompañan a un proceso productivo. El problema surge con la inestabilidad del mundo laboral y la itinerancia en los puestos de trabajo, situaciones que se agudizan en el área de Arquitectura e Ingeniería, supeditada a innegables avances científicos y tecnológicos, lo que convierte a los profesionales de este sector en estudiosos durante toda su vida profesional, para responder con eficacia y eficiencia a los problemas que se les presenten. De la misma manera, es necesario que los alumnos se formen para trabajar en equipos multidisciplinares. Diferentes autores enfatizan en la necesidad de formar a los ingenieros para que sean capaces de dar soluciones viables, eficientes y rápidas en el desempeño profesional.

Como ya se ha indicado la identificación de los estilos de aprendizaje de los alumnos, es importante en la medida que permite conocer cómo estos aprenden. Sin embargo, esta fase de diagnóstico es solo el comienzo del plan de actuación, plan que deberá promover, desarrollar e implementar estrategias didácticas que permitan el desarrollo de todos y cada uno de los estilos, adaptando el contexto educativo convencional en otro flexible e interactivo con las peculiaridades e intereses individuales (Ventura et al., 2014), máxime en el área científico-técnica.

Los alcances que se deben destacar de esta investigación son, de una parte, la aportación a la identificación de los estilos de aprendizaje que presentan los alumnos estudiados y la influencia del sexo en el estilo de aprendizaje, lo que supone un avance importante para readaptar las prácticas de aula, de manera que todos los alumnos puedan desarrollarse intelectualmente de la manera más completa posible. De otra parte, se ha puesto de manifiesto empíricamente que, por lo general, los alumnos tienden a sobrevalorar los conocimientos que poseen. E igualmente importante, conocer que el estilo de aprendizaje condiciona el éxito en determinadas asignaturas.

Es conveniente plantear tres limitaciones a la presente investigación, que al mismo tiempo se convierten en un avance de líneas de futuro al estudio presentado. Como primera limitación el tamaño de la muestra, que si bien aporta datos relevantes para una toma inicial de decisiones, no permite la generalización de resultados, por lo que sería oportuno y necesario replicar el estudio en cursos sucesivos. La segunda limitación es la escasa representatividad del estilo teórico, lo que obliga a continuar esta investigación con muestras de mayor tamaño que permitan conseguir una mayor frecuencia de alumnos con este estilo. Y la 
tercera, la carencia de estudios similares en el ámbito español, lo que hace necesario su generalización en el resto de Escuelas, para poder llegar a resultados lo más extrapolables y generalizables posible, máxime si se tiene en cuenta las importantes posibilidades que tienen los alumnos de estudiar en centros distintos al propio, como pueden ser los programas Sócrates, Erasmus, entre otros.

Continuando con las líneas de futuro, sería conveniente no solo replicar el estudio en el propio centro y generalizarlo a otras Escuelas de la titulación, sino también incorporar al estudio el diagnóstico de los enfoques y estrategias de aprendizaje y de motivación que presentan los alumnos. Y de la misma manera, para completar de manera integral el estudio, sería importante diagnosticar el estudio de enseñanza de los docentes, y estudiar la influencia que estos ejercen sobre el estilo de aprendizaje de los alumnos.

Derivado de esta investigación se pueden proponer tres líneas de intervención. La primera concienciar a los profesores del Centro de la necesidad de tomar en consideración los estilos de aprendizaje de los alumnos, para lo que será necesario planes de formación específico en técnicas didácticas y/o la colaboración de expertos en educación universitaria que actúen como asesores. La segunda trabajar directamente con los alumnos para que reconozcan sus estilos de aprendizaje, y comprendan la necesidad de desarrollar características de los otros estilos, lo que se podría resolver con cursos de orientación al estudio al comienzo del primer semestre. Estas dos líneas son acordes al conocimiento y mejoramiento de los estilos de aprendizaje. Sin embargo, es igualmente necesario paliar el bajo nivel de conocimiento en materias básicas que condicionan el éxito académico de los alumnos, y para ello se podría recurrir a la implantación de los cursos 0 , o de iniciación, en los que se impartan los conocimientos necesarios para que los alumnos puedan hacer un seguimiento correcto de las asignaturas que deben cursar. Entendiéndose que estas tres líneas desarrolladas simultáneamente conducirían de una parte a ajustar los métodos a los estilos de los alumnos, a que estos sean conscientes de que deben desarrollar estrategias de estudio adecuadas, disminuir el porcentaje de deserción y retraso en los estudios y aumentar la tasa de rendimiento.

Y cabría preguntarse ¿después que hay?, ya se ha realizado el diagnóstico, ¿y, ahora? ¿Se puede planificar y utilizar conscientemente actividades, prácticas de aula,..., en los que se tengan en cuenta todos los estilos de aprendizaje, para no favorecer a los alumnos de un estilo concreto? Dado que en la investigación realizada se ha utilizado el cuestionario CHAEA, las actuaciones vendrán condicionadas por el sustento teórico del mismo, así se deben planificar actividades en las que intervengan las cuatro fases del aprendizaje: activa, reflexiva, teórica y pragmática. De acuerdo con Alonso et al. (2012) lo ideal sería que todos los alumnos fueran capaces de experimentar, reflexionar, elaborar hipótesis y aplicar a partes iguales, situación en la que los individuos se encontrarían en equilibrio. Por ello, las actividades que se planteen estarán preferentemente basadas en estudios de casos, aprendizaje basado en problemas, aprendizaje basado en proyectos, que obligan a que los alumnos indaguen, analicen, sinteticen y elaboren propuestas.

Lo recomendable es comenzar por actividades sencillas y cortas, de manera que los alumnos se vayan involucrando en sus procesos personales de mejora del aprendizaje, y adquiriendo confianza en sus posibilidades. Si las actividades se van a desarrollar en grupo, sería interesante que fuera el profesor quien atribuyera las funciones y roles que deben desempeñar cada alumno, evitando de esta manera que se asignen aquellos que saben que no les va a resultar problemático desarrollar, en los que se van a sentir más cómodos y les va a suponer menos esfuerzo. De la misma manera, y dado que se va a forzar a los alumnos a realizar tareas en las que no se sentirán inicialmente cómodos, el profesor deberá supervisar no solo el resultado final del trabajo, sino todo el proceso seguido por los alumnos, acompañándolos en el trayecto creativo con asesoramiento, planteándoles estrategias alternativas si fuera menester, motivarlos al valorar el esfuerzo que están realizando y plantear las críticas, comentarios o sugerencias que deba hacer de forma constructiva.

\section{CONCLUSIONES}

A partir del análisis de los resultados obtenidos, así como de la discusión planteada se extraen las siguientes conclusiones en la investigación realizada:

a) La secuencia de estilos de aprendizaje dominante ordenados de mayor a menor frecuencia es: Reflexivo, Pragmático, Activo y Teórico, mientras que el orden de mayor a menor puntuación en las escalas de los estilos ha sido: Reflexivo, Pragmático, Teórico y Activo.

b) El estilo de aprendizaje dominante del CHAEA influye, solo y de diversa forma en las calificaciones obtenidas en la asignatura "Materiales I", donde destacan con la media más alta los alumnos de estilo reflexivo, en la asignatura "Fundamentos físicos" donde sobresalen los alumnos pragmáticos, y en la asignatura "Estructuras I" donde destacan los alumnos reflexivos y pragmáticos. 
c) No existe paralelismo entre lo que el alumno considera que sabe y la calificación que obtiene.

d) Hay relación en los resultados obtenidos por los alumnos en las asignaturas pertenecientes a una misma área de conocimiento (construcciones arquitectónicas, expresión gráfica, y científico-tecnológica), es decir, los alumnos que obtienen calificaciones altas en una asignatura de un área de conocimiento lo hacen también en el resto de asignaturas del mismo área, y viceversa, los alumnos que obtienen calificaciones bajas en una asignatura de un área de conocimiento también tienen notas bajas en el resto de asignaturas del mismo área de conocimiento.

\section{REFERENCIAS}

Abdul-Raman, S. y B. Boulay, Learning programming via worked-examples: Relation of learning styles to cognitive load, doi:10.1016/j.chb.2013.09.007, Computers in Human Behavior, 30(1): 286-298 (2014)

Acevedo, C. G. y F. Rocha, Estilos de aprendizaje, género y rendimiento académico, Revista Estilos de Aprendizaje: 8(4), 71-84 (2011)

Acevedo, D., Sh. Cavadia y A. Alvis, Estilos de aprendizaje de los Estudiantes de la Facultad de Ingeniería de la Universidad de Cartagena (Colombia), doi: 10.4067/S0718-50062015000400003, Formación Universitaria, 8(4), 15-22 (2015a)

Acevedo, D., D. Tirado y P. Montero, Perfil de Aprendizaje y Rendimiento Académico en una Asignatura de Química en Modalidad a Distancia y Presencial en dos Programas de Ingeniería, doi: 10.4067/S071850062015000600006, Formación Universitaria: 8(6), 39-46 (2015b)

Alonso, C., D. Gallego y P. Honey. Los estilos de aprendizaje: Procedimientos de diagnóstico y mejora, Ediciones Mensajero, Bilbao, España (2012)

Arias, W. L., J. Zegarra y O. Justo, Estilos de aprendizaje y metacognición en estudiantes de psicología de Arequipa, Liberabit: 20(2), 267-279 (2014)

Bolívar, J.M. y F., Rojas, Los estilos de aprendizaje y el Locus de control en estudiantes que inician estudios superiores y su vinculación con el rendimiento académico, Investigación y Postgrado: 23(3), 199-215 (2008)

Cantú, I. L., El estilo de aprendizaje y la relación con el desempeño académico de los estudiantes de Arquitectura de la UANL, Ciencia UANL: VII(1), 72-79 (2004)

Craveri, A. M. \& M. Anido, El aprendizaje de Matemática con herramienta computacional en el marco de la teoría de los estilos de aprendizaje, Revista de Estilos de Aprendizaje: 3(3), 102-123 (2009)

Demirbas, O. y $\mathrm{H}$. Demirkan, Learning styles of design students and the relationship of academic performance and gender in design education, doi:10.1016/j.learninstruc.2007.02.007, Learning and Instruction: 17, 345-359 (2007)

Escalante, E., R. Barrionuevo y M. Mercado, Aplicación de técnicas multidimensionales al estudio de los estilos de aprendizaje en estudiantes de ingeniería de la Universidad Tecnológica Nacional de Mendoza y La Rioja, Cognición: 5(21), 34-42 (2009)

Felder R. y L. Silverman, Learning and Teaching Styles, Engineering Education: 78(7), 674-681 (1988)

Gallego, D.J. y A. Nevot, Los estilos de aprendizaje y la enseñanza de las matemáticas, Revista Complutense de Educación: 19(1) (2008)

Gentry, J. A. y M. G. Helgesen, Using Learning Style Information to Improve the Core Financial Management Course, Financial Practice and Education: Spring-Summer (1999)

George, D., y P. Mallery, SPSS/PC+ step by step: a simple guide and reference, Wadsworth Publishing Company, Belmont, USA (1995)

Gil Madrona, P., O. Contreras, y J. C. Pastor, Estilos de aprendizaje de los estudiantes de magisterio: Especial consideración de los alumnos de educación física: Revista de currículum y formación del profesorado (2007). http://www.ugr.es/local/recfpro/rev112ART8.pdf. Acceso enero (2008)

Gravini, M., Estilos de aprendizaje de los estudiantes de primer semestre de los programas de Psicología e Ingeniería Industrial de la Universidad Simón Bolívar de Barranquilla, Psicogente: 11(9), 24-33 (2008)

Hamidah, J. S., M. N. Sarina y J. Kamaruzaman, The Social Interaction Learning Styles of Science and Social Science Students, dx.doi.org/10.5539/ass.v5n7p58, Asian Social Science: 5(7), 58-64 (2009)

Holvikivi, J., Learning styles in engineering education: the quest to improve didactic practice, doi: 10.1080/03043790701332909, European Journal of Engineering Education: 32(4), 401-408 (2007) 
Kolb, A. y D. A. Kolb, Learning Styles and Learning Spaces: Enhancing Experiential Learning in Higher Education, Academy of Management Learning \& Education: 4(2), 193-212 (2005)

Komarraju, M., y otros tres autores, The big five personality traits, learning styles, and academic achievement, doi:10.1016/j.paid.2011.04.019, Personality and Individual Differences: 51, $472-477$ (2011)

Luengo, R., y J. J. González, Relación entre los estilos de aprendizaje, el rendimiento en matemáticas y la elección de asignaturas optativas en alumnos de ESO, Relieve: 11(2) (2005). http://www.uv.es/RELIEVE/v11n2/RELIEVEv11n2_4.htm. Acceso: enero (2006)

Morales-Ramírez, A., y otros cuatro autores, Estilos de aprendizaje en estudiantes universitarios de ingeniería en computación e informática administrativa, Revista Estilos de Aprendizaje: 9(5), 156-168 (2012)

Moreno, C., Y. Molina y J. A. Chacón, Impacto del estilo pedagógico integrador en los estudiantes de licenciatura en educación básica de la facultad de estudios a distancia, doi: 10.4067/S071850062014000600005, Formación Universitaria: 7(6), 37-44 (2014)

Orhun, N., The relationship between learning styles and achievement in calculus course for engineering students, doi:10.1016/j.sbspro.2012.06.710, Procedia Social and Behavioral Sciences: 46, 638-642 (2012)

Ramos, G. E. y M. L. Triana, Diagnóstico del proceso enseñanza y su relación con los estilos y estrategias de aprendizaje de los estudiantes del programa de ingeniería industrial de la Universidad Tecnológica, Scientia Et Technica: XVII(47), 270-275 (2011)

Safarin, M., y otros tres autores: Visualization Skills and Learning Style Patterms among Engineering Students at Universiti Teknologi Malaysia, doi:10.1016/j.sbspro.2013.10.114, Procedia-Social and Behavorial Science: 93(2), 1769-1775 (2013)

Ventura, A. C., El ajuste instructivo entre estilos de aprendizaje y enseñanza en la universidad, Revista de Psicología: 31(2), 265- 286 (2013)

Ventura, A. C., y tres autores más, Estilos de aprendizaje y enseñanza de Ingeniería: una propuesta de educación adaptativa para primer año, Educación en Ingeniería: 9(18), 178-189 (2014)

Villamizar, G., Estilos de aprendizaje y rendimiento académico en estudiantes de ingeniería civil, Informes Psicológicos: 12(2), 41-54 (2012) 